\title{
Multi-Locus Genome-Wide Association Study and Genomic Selection of Kernel Moisture Content at the Harvest Stage in Maize
}

\author{
Guangfei Zhou ${ }^{1,2}$, Qiuli Zhu ${ }^{3}$, Yuxiang Mao ${ }^{1}$, Guoqing Chen ${ }^{1,2}$, Lin Xue ${ }^{1,2}$, Huhua Lu ${ }^{1}$, \\ Mingliang Shi ${ }^{1}$, Zhenliang Zhang ${ }^{1}$, Xudong Song ${ }^{1}$, Huimin Zhang ${ }^{1}$ and Derong Hao ${ }^{1 *}$ \\ 1 Department of Food Crops, Jiangsu Yanjiang Institute of Agricultural Science, Nantong, China, ${ }^{2}$ Jiangsu Collaborative \\ Innovation Centre for Modern Crop Production, Nanjing, China, ${ }^{3}$ Jiangsu Nantong Crop Cultivation Technique Direction \\ Station, Nantong, China
}

OPEN ACCESS

Edited by:

Yongzhong Xing,

Huazhong Agricultural

University, China

Reviewed by:

Jianbo He

Nanjing Agricultural University, China

Yingjie Xiao,

Huazhong Agricultural

University, China

*Correspondence:

Derong Hao

drhao2008@163.com

Specialty section

This article was submitted to

Plant Breeding,

a section of the journal

Frontiers in Plant Science

Received: 20 April 2021

Accepted: 16 June 2021

Published: 09 July 2021

Citation:

Zhou G, Zhu Q, Mao Y, Chen G, Xue L, Lu H, Shi M, Zhang Z, Song $X$,

Zhang $H$ and Hao D (2021)

Multi-Locus Genome-Wide

Association Study and Genomic

Selection of Kernel Moisture Content at the Harvest Stage in Maize.

Front. Plant Sci. 12:697688.

doi: 10.3389/fpls.2021.697688
Kernel moisture content at the harvest stage $(\mathrm{KMC})$ is an important trait that affects the mechanical harvesting of maize grain, and the identification of genetic loci for KMC is beneficial for maize molecular breeding. In this study, we performed a multi-locus genome-wide association study (ML-GWAS) to identify quantitative trait nucleotides (QTNs) for KMC using an association mapping panel of 251 maize inbred lines that were genotyped with an Affymetrix CGMB56K SNP Array and phenotypically evaluated in three environments. Ninety-eight QTNs for KMC were detected using six ML-GWAS models (mrMLM, FASTmrMLM, FASTmrEMMA, PLARmEB, PKWmEB, and ISIS EM-BLASSO). Eleven of these QTNs were considered to be stable, as they were detected by at least four ML-GWAS models under a uniformed environment or in at least two environments and BLUP using the same ML-GWAS model. With qKMC5.6 removed, the remaining 10 stable QTNs explained $<10 \%$ of the phenotypic variation, suggesting that KMC is mainly controlled by multiple minor-effect genetic loci. A total of 63 candidate genes were predicted from the 11 stable QTNs, and 10 candidate genes were highly expressed in the kernel at different time points after pollination. High prediction accuracy was achieved when the KMC-associated QTNs were included as fixed effects in genomic selection, and the best strategy was to integrate all KMC QTNs identified by all six ML-GWAS models. These results further our understanding of the genetic architecture of KMC and highlight the potential of genomic selection for KMC in maize breeding.

Keywords: maize (Zea mays L), kernel moisture content, multi-locus genome-wide association study, quantitative trait nucleotide, candidate gene, genomic selection

\section{INTRODUCTION}

Kernel moisture content at the harvest stage (KMC) is one of the important traits that influence maize mechanical harvesting, especially in high latitude areas (Sala et al., 2012; Li et al., 2017). Since the 1970s, many developed countries, such as the United States and Germany, have achieved fully mechanical harvesting of maize. By contrast, other countries, like China, have not yet implemented mechanical harvesting, primarily due to a lack of suitable maize varieties (Liu et al., 2013). The high KMC of currently used maize varieties restricts mechanical harvesting and represents the major barrier to maize development in China (Zhou et al., 2016, 2020; Li et al., 2017). Therefore, the 
genetic improvement of $\mathrm{KMC}$ and the breeding of elite varieties with low KMC is a major goal for maize breeders in China.

The loss of maize kernel moisture occurs in two phases. The first phase lasts from pollination to kernel physiological maturity. During this phase, water in the kernel is replaced with carbohydrates, oils, proteins, etc., and the moisture of the kernel is highly dependent on its own physiological characteristics; this is called the physiological dehydration stage. The second phase lasts from physiological maturity to harvest. During this phase, the change in kernel moisture is primarily caused by moisture evaporation into the air and is thus readily influenced by environmental factors and other agronomic traits; this is known as the field dehydration stage (Brooking, 1990; Reid et al., 2010). Rapid rates of kernel filling and field dehydration are marked features of maize varieties with low KMC (Johnson and Tanner, 1972; Sala et al., 2006). Fewer husk layers as well as shorter and lighter husks are associated with greater loss of kernel moisture after physiological maturity (Reid et al., 2010; Li et al., 2016; Zhou et al., 2018). In addition, moisture from the kernels can be transported to other plant parts through the cob and stem in response to water potential differences (Zhou et al., 2018).

Previous studies have revealed that KMC is controlled by numerous quantitative trait loci (QTLs), and hundreds of QTLs for maize KMC have been identified (Beavis et al., 1994; Melchinger et al., 1998; Austin et al., 2000; Ho et al., 2002; Mihaljevic et al., 2004, 2005; Blanc et al., 2006; Sala et al., 2006; Frascaroli et al., 2007; Capelle et al., 2010; Kebede et al., 2016; Song et al., 2017; Zhou et al., 2018; Liu et al., 2020; Yin et al., 2020b; Zhang et al., 2020; Li et al., 2021). Using meta-analysis, 44 and 34 meta-QTLs for KMC were identified by Xiang et al. (2012) and Sala et al. (2012), respectively. Liu et al. (2020) narrowed a major QTL for KMC ( $q$ Gwc1.1) to a $2.05-\mathrm{Mb}$ genomic region on chromosome 1 using a recombinant-derived progeny test. Li et al. (2021) cloned a gene (gar2-related nucleolar protein, GAR2) for KMC on maize chromosome 7. Yin et al. (2020b) identified seven QTLs for KMC using multiple-environment analysis and revealed that the interactions between QTLs and the environment were larger than their additive effects. Zhou et al. (2018) detected five QTLs for KMC through a mixed linear model (MLM) of single-locus genome-wide association study (SL-GWAS).

The Bonferroni correction for multiple tests is frequently used in SL-GWAS to reduce spurious associations, and this results in the elimination of some positive loci with small effects. Multilocus GWAS (ML-GWAS), an alternative GWAS method, was developed to address this issue; it considers the information from all markers simultaneously and does not require a multiple testing correction. ML-GWAS has been shown to have higher power and accuracy for the detection of quantitative trait nucleotides (QTNs) in maize. Zhang et al. (2018) used four ML-GWAS methods (mrMLM, FASTmrEMMA, ISIS EMBLASSO, and pLARmEB) to identify QTNs for three stalk lodging resistance-related traits in maize and reported that the methods were reliable and complementary. Xu et al. (2018) compared one SL-GWAS method (GEMMA) and three MLGWAS methods (FASTmrEMMA, FarmCPU, and LASSO) for the genetic detection of maize starch pasting properties, and more QTNs were detected by individual ML-GWAS methods than by the SL-GWAS method. An et al. (2020) used one SLGWAS method (MLM) and six ML-GWAS methods (mrMLM, FASTmrMLM, FASTmrEMMA, pLARmEB, pKWmEB, and ISIS EMBLASSO) to dissect the genetic architecture of maize kernel row number. The largest number of QTNs were identified with the mrMLM method, and the most co-detected QTNs were identified with ISIS EM-BLASSO.

Given the lack of large-effect QTLs, the use of markerassisted selection (MAS) for KMC is not ideal in maize breeding programs, and it is necessary to incorporate far more markers. Genomic selection (GS), an upgraded form of MAS, aims to use genetic effects of genome-wide molecular markers to estimate the genomic estimated breeding value (GEBV) of individuals based on optimum statistical models (Meuwissen et al., 2001). This approach has been considered most promising for the genetic improvement of complex traits controlled by multiple genes with minor effects (Wang X. et al., 2018; Xu et al., 2020). Controlling costs by using fewer markers while still achieving accurate predictions for complex quantitative traits remains a challenge (Xu et al., 2020). Recently, several studies reported that taking association markers for interesting traits detected by GWAS into account and including them as fixed effects in GS models resulted in higher accuracy than that achieved with GS models using genome-wide markers (Spindel et al., 2016; Qin et al., 2019; Ravelombola et al., 2019; An et al., 2020; Sehgal et al., 2020).

In this study, we used 251 maize inbred lines that were genotyped using an Affymetrix CGMB56K SNP Array and phenotypically evaluated in three field trials to (i) identify significant QTNs for KMC using ML-GWAS, (ii) predict candidate genes associated with KMC, and (iii) explore the potential of GS for KMC in maize.

\section{MATERIALS AND METHODS \\ Plant Material and Field Experiments}

An association mapping panel of 251 diverse maize inbred lines was used as the plant material in this study (Supplementary Table 1).

The field experiments were performed in three environments in 2020: Nantong, Jiangsu Province (NT, $120^{\circ} \mathrm{E}, 31^{\circ} \mathrm{N}$ ), which is in mid-eastern China and has an average temperature of $15.1^{\circ} \mathrm{C}$ and an average rainfall of $1,040 \mathrm{~mm}$ per year; Xinxiang, Henan Province $\left(\mathrm{XX}, 113^{\circ} \mathrm{E}, 35^{\circ} \mathrm{N}\right)$, which is in the middle of China and has an average temperature of $15.5^{\circ} \mathrm{C}$ and an average rainfall of $573.4 \mathrm{~mm}$ per year; and Sanya, Hainan Province (SY, $\left.108^{\circ} \mathrm{E}, 18^{\circ} \mathrm{N}\right)$, which is in southern China and has an average temperature of $25.7^{\circ} \mathrm{C}$ and an average rainfall of $1,347 \mathrm{~mm}$ per year. Each line was grown in single rows, which were $3 \mathrm{~m}$ in length and spaced $0.6 \mathrm{~m}$ apart, thereby giving a planting density of 65,000 plants/ha. The trial followed a randomized complete block design with two replicates per environment. The agronomic management of the field experiments was the same in the three environments. 


\section{Phenotypic Evaluation and Data Analysis}

As described in our previous study (Zhou et al., 2018), before the experimental treatment, the physiological maturity of each line was evaluated in the field. According to their growth periods, the 251 maize inbred lines were sown on three separate dates to obtain similar physiological maturity in each environment (Supplementary Table 1). In NT, they were planted on the 22nd, 26th, and 30th of March, and the harvest stage was adjusted to between the 24th and 26th of July. In XX, they were planted on the 1st, 5th, and 9th of June, and the harvest stage was adjusted to between the 26th and 28th of September. In SY, they were planted on the 14th, 18th, and 22nd of November, and the harvest stage was adjusted to between the 5th and 7th of the following March. The ears were bagged before silking, and artificial pollination was performed at the same time for each line. The KMC for 68 uniformly growing plants in the middle of rows was measured using a hand-held moisture meter. At 10 days after physiological maturity, the KMC of each plant was recorded one time at the middle part of the ear.

The phenotypic data were analyzed using $\mathrm{R}$ version 3.6.3 for Windows (https://www.r-project.org/). Analysis of variance (ANOVA) of KMC was performed using lmer function of the lme4 package based on the following model: $y_{i j k}=\mu+G_{i}+E_{j}$ $+G E_{i j}+R_{j k}+e_{i j k}$, where $y_{i j k}$ is the KMC on the $i^{\text {th }}$ genotype in the $j^{\text {th }}$ environment and $k^{\text {th }}$ replication, $\mu$ is the grand mean over all environments, $G_{i}$ is the genotypic effect of the $i^{\text {th }}$ genotype, $E_{j}$ is the environmental effect of the $j^{\text {th }}$ environment, $G E_{\mathrm{ij}}$ is the genotype $\times$ environment interaction effect of the $i^{\text {th }}$ genotype and $j^{\text {th }}$ environment, $R_{j k}$ is the effect of the $k^{\text {th }}$ replication in $j^{\text {th }}$ environment, and $e_{i j k}$ is the residual error. The trait heritability $\left(H^{2}\right)$ was estimated following Knapp et al. (1985): the individual environment $H^{2}(\%)=\sigma_{\mathrm{g}}^{2} /\left(\sigma_{\mathrm{g}}^{2}+\sigma_{\mathrm{e}}^{2} / r\right) \times 100 \%$, and the multiple environments $H^{2}(\%)=\sigma_{\mathrm{g}}^{2} /\left(\sigma_{\mathrm{g}}^{2}+\sigma_{\mathrm{ge}}^{2} / n+\sigma_{\mathrm{e}}^{2} / n r\right) \times 100 \%$, where $\sigma_{\mathrm{g}}^{2}$ is the genotypic variance, $\sigma_{\mathrm{ge}}^{2}$ is the variance for the interaction of genotype with environment, $\sigma_{\mathrm{e}}^{2}$ is the error variance, $n$ is the number of environments, and $r$ is the number of replications. To minimize the effects of the environment, the best linear unbiased prediction (BLUP) for KMC across the three environments was estimated using the lmer function of the lme4 package with the same ANOVA model. The normal distribution test (W-value) of KMC in each environment was estimated using the Shapiro.test function of the stats package.

\section{Genotyping, Population Structure, Linkage Disequilibrium, and Relative Kinship}

Genotypes of the 251 maize inbred lines were evaluated using an Affymetrix CGMB56K SNP Array, which contains 56,000 single nucleotide polymorphisms (SNPs) and is made by China Golden Marker (Beijing) Biotech Co., Beijing, China. After quality control, 32,853 SNPs with minor allele frequencies $>5 \%$ and missing data $<20 \%$ were used for subsequent analysis. The genotypic data can be downloaded from the website https://pan. baidu.com/s/1_V0fm7hsxNdMbyYzciQsWg.

Population structure was assessed using STRUCTURE 2.3 (Pritchard et al., 2000). The number of subpopulations $(K)$ was set from 1 to 10 with five independent runs for each $K$. Both burnin periods and Markov chain Monte Carlo replication number were set at 100,000 in each run under the admixture model. The $K$ value was estimated by the log likelihood of the data $[\mathrm{LnP}(\mathrm{D})]$ and an ad hoc statistic $\Delta \mathrm{K}$, based on the rate of change of $\operatorname{LnP}(\mathrm{D})$ between successive $K$ values (Evanno et al., 2005). Nei's genetic distance (Nei, 1972) was calculated and used to construct a neighbor-joining tree with MEGA-X software (Kumar et al., 2018). The linkage disequilibrium (LD) parameter $r^{2}$ between pairwise SNPs was calculated with PLINK (Purcell et al., 2007), which window size was set at $1,000 \mathrm{~kb}$ and $r^{2}$ was set at 0.2 . The relative kinship matrix of the 251 lines was computed using SPAGeDi 1.3 (Hardy and Vekemans, 2002) with negative values between two individuals set to zero.

\section{Multi-Locus Genome-Wide Association Study}

ML-GWAS was conducted using the mrMLM package (https://cran.r-project.org/web/packages/mrMLM/index.

html), including six statistical models: mrMLM (Wang et al., 2016), FASTmrMLM (Tamba and Zhang, 2018), FASTmrEMMA (Wen et al., 2018), pLARmEB (Zhang et al., 2017), pKWmEB (Ren et al., 2018), and ISIS EM-BLASSO (Tamba et al., 2017). The mrMLM is a multi-locus model including markers selected from the random-SNP-effect MLM with a less stringent selection criterion (Wang et al., 2016). The FASTmrMLM is relatively faster with higher statistical power and accuracy in estimating QTNs as compared to mrMLM (Tamba and Zhang, 2018). The FASTmrEMMA combines the MLM and the expectation maximization empirical Bayes method. The pLARmEB integrates least angle regression with empirical Bayes (Zhang et al., 2017), while the pKWmEB integrates Kruskal-Wallis test with empirical Bayes (Ren et al., 2018). The ISIS EMBLASSO can detect significant associations with highest robustness and accuracy as compared to mrMLM and FASTmrEMMA (Tamba et al., 2017). Default values were used for all parameters, and the threshold of logarithm of odds (LOD) $\geq 3$ (or $P \leq 0.0002$ ) was selected to determine significant QTNs (Zhang et al., 2019). To confirm the efficiency of ML-GWAS, one widely used SL-GWAS method, MLM, was conducted using TASSEL 5.0 (Bradbury et al., 2007), controlling for population structure and kinship. The threshold was also set at $P \leq 0.0002\left[-\log _{10}(P) \geq 3.70\right]$.

Two types of QTNs were defined as stable QTNs. One is model-stable QTN (msQTN), which is identified by at least four ML-GWAS models under a uniformed environment, another is environment-stable QTN (esQTN), which is identified by in at least two environments and BLUP using the same MLGWAS model.

\section{Candidate Gene Analysis}

Based on the B73 reference genome v4 (https://www.maizegdb. org/gbrowse), the available genes within regions from $100 \mathrm{~kb}$ upstream to $100 \mathrm{~kb}$ downstream (LD of the association mapping panel) around the stable QTNs were regards as candidates. Candidate gene annotation was performed at NCBI (https:// www.ncbi.nlm.nih.gov/). Expression data for candidate genes were collected from qTeller (https://qteller.maizegdb.org/). 


\section{Genomic Selection}

GS was performed using the ridge regression best linear unbiased predictor (rrBLUP) model (Meuwissen et al., 2001) in rrBLUP package of $\mathrm{R}$ software (Endelman, 2011). The rrBLUP model (model 1) was: $y_{i}=\mu+\sum_{k=1}^{p} x_{i k} \beta_{k}+\varepsilon_{i}$, where $y_{i}$ is the predicted phenotype of $i^{\text {th }}$ individual, $\mu$ is the grand mean, $x_{i k}$ is the genotype at the $k^{\text {th }}$ marker of the $i^{\text {th }}$ individual, $p$ is the total number of markers (i.e., 32,853 high quality markers from the Affymetrix array), $\beta_{k}$ is the estimated random additive marker effect of the $k^{\text {th }}$ marker, and $\varepsilon_{i}$ is the residual error. To improve the prediction accuracy, the significant markers identified by MLGWAS were included as fixed effects in the following model (model 2): $y_{i}=\mu+\sum_{j=1}^{m} x_{i k} \alpha_{j}+\sum_{k=1}^{p} x_{i k} \beta_{k}+\varepsilon_{i}$, where $m$ is the number of significant markers identified ML-GWAS considered as fixed effect covariates, $\alpha_{j}$ is the fixed additive effect of the $j^{\text {th }}$ marker, and the remaining terms are the same as those described in model 1. Twelve sets of markers were included as fixed effects in model 2: the significant markers identified by mrMLM, FASTmrMLM, FASTmrEMMA, pLARmEB, pKWmEB, and ISIS EM-BLASSO; the markers identified by at least two, three, four, five, or six models; and all significant markers identified by all six models.

Prediction accuracy was evaluated using 5-fold cross validation with 100 iterations. In brief, the association panel was randomly divided into five equal subsets; four subsets were regarded as the training population, and the remaining set was considered to be the testing population. The prediction accuracy was defined as the coefficient of determination between the predicted and the observed values based on the linear regression analysis.

\section{RESULTS}

\section{Population Structure, Linkage Disequilibrium, and Relative Kinship}

STRUCTURE software was used to calculate the Bayesian clustering from $K=1$ to 10 with five independent runs for each $K$. The $\operatorname{LnP}(\mathrm{D})$ values increased as $K$ increased from 1 to 10 without an obvious inflection point, and $\Delta K$ reached its peak at $K$ $=6$ (Figure 1A), suggesting that this association mapping panel could be divided into six subgroups (Figure 1B). A neighborjoining tree was constructed based on Nei's genetic distance, and it showed six main clusters for this panel (Figure 1C), consistent with the STRUCTURE results.

The six subpopulations were designated PA, PB, Tangsipingtou (TSPT), Lancaster (LAN), BSSS, and X (Supplementary Table 1). Subgroup PA, including 45 inbred lines, tended to be improved Reid lines, such as Zheng 58 and Ye478. Subgroup PB, including 45 inbred lines, derived mainly from hybrid 78599 and included Qi319, ND1145, etc. Subgroup TSPT, including 74 inbred lines, consisted mainly of inbred lines such as Huangzaosi and Chang7-2. Subgroup LAN, including 15 inbred lines, contained the representative inbred line Mo17. Subgroup BSSS derived from the synthetic variety BSSS and included 44 inbred lines; its representative inbred line was B73.
Subgroup X, including 28 inbred lines, derived mainly from hybrid Xianyu335 that is widely cultivated in China.

LD decayed differently in the 10 chromosomes; chromosome 7 had the most rapid decay rate, and chromosome 4 had the slowest. The average LD decay distance across all chromosomes was $\sim 100 \mathrm{~kb}$, where the LD parameter $\left(r^{2}\right)$ dropped to half of its maximum value (Figure 2A). The average pairwise relative kinship value was 0.076 . Pairwise relative kinship values of 0 accounted for $64.64 \%$ of all kinship values, values from 0 to 0.2 accounted for $88.03 \%$, and values $>0.5$ accounted for only $4.07 \%$ (Figure 2B). This result revealed that the 251 inbred lines used in this study were distantly related.

\section{Phenotypic Variation in KMC}

Descriptive statistics for KMC are presented in Table 1. KMC showed abundant variation among the 251 lines in each environment and was positively correlated among the different environments (Figure 3). The KMC in each environment approximately fitted a normal distribution with small skewness and kurtosis and high $\mathrm{W}$-value $(P>0.05)$ (Table 1; Figure 3), suggesting that KMC was controlled by multiple genetic loci in this association mapping panel. ANOVA showed that the environment exerted significant influence on KMC (Supplementary Table 2), and the heritability was high (67.3675.86\%) (Table 1).

Based on the BLUP values across the three environments, 11 of the 251 lines showed low KMC (below 27\% moisture) (ID: $110,111,114,131,188,209,235,242,246,247$, and 249), and these are marked in bold in Supplementary Table 1. Fifty-five lines showed high KMC (above 40\%), and the remaining lines showed moderate KMC (Supplementary Table 1). There was a significant difference in KMC among the six subpopulations; the KMC of subgroup X was the lowest, and that of subgroup PA was the highest (Figure 4A).

\section{Multi-Locus Genome-Wide Association Study of KMC}

A total of 98 QTNs were detected in NT, XX, SY, and BLUP across all environments by the six ML-GWAS models, and each explained $0.67-26.96 \%$ of the phenotypic variation in KMC (Supplementary Table 3). Thirty-eight, 35, 23, 27, 34, and 39 QTNs were detected by mrMLM (Supplementary Figure 1), FASTmrMLM (Supplementary Figure 2), FASTmrEMMA (Supplementary Figure 3), PLARmEB, PKWmEB (Supplementary Figure 4), and ISIS EM-BLASSO, respectively. However, only 7 QTNs were detected in NT, XX, SY, and BLUP across all environments by the SL-GWAS model (MLM) (Supplementary Figure 5; Supplementary Table 3). Expect for $q K M C 7.4$, the remaining 6 QTNs were overlapped with those from ML-GWAS models.

Among 98 QTNs, 44, 27, 16, 7, and 4 QTNs were co-detected by at least two, three, four, five, or six ML-GWAS models, respectively. 25, 25, 32, and 42 were detected in NT, XX, SY, and BLUP, respectively. However, no QTN was detected in three environments and BLUP, and only three QTNs ( $q K M C 2.15$, $q K M C 6.1$, and $q K M C 8.2$ ) were detected in two environments and BLUP. 

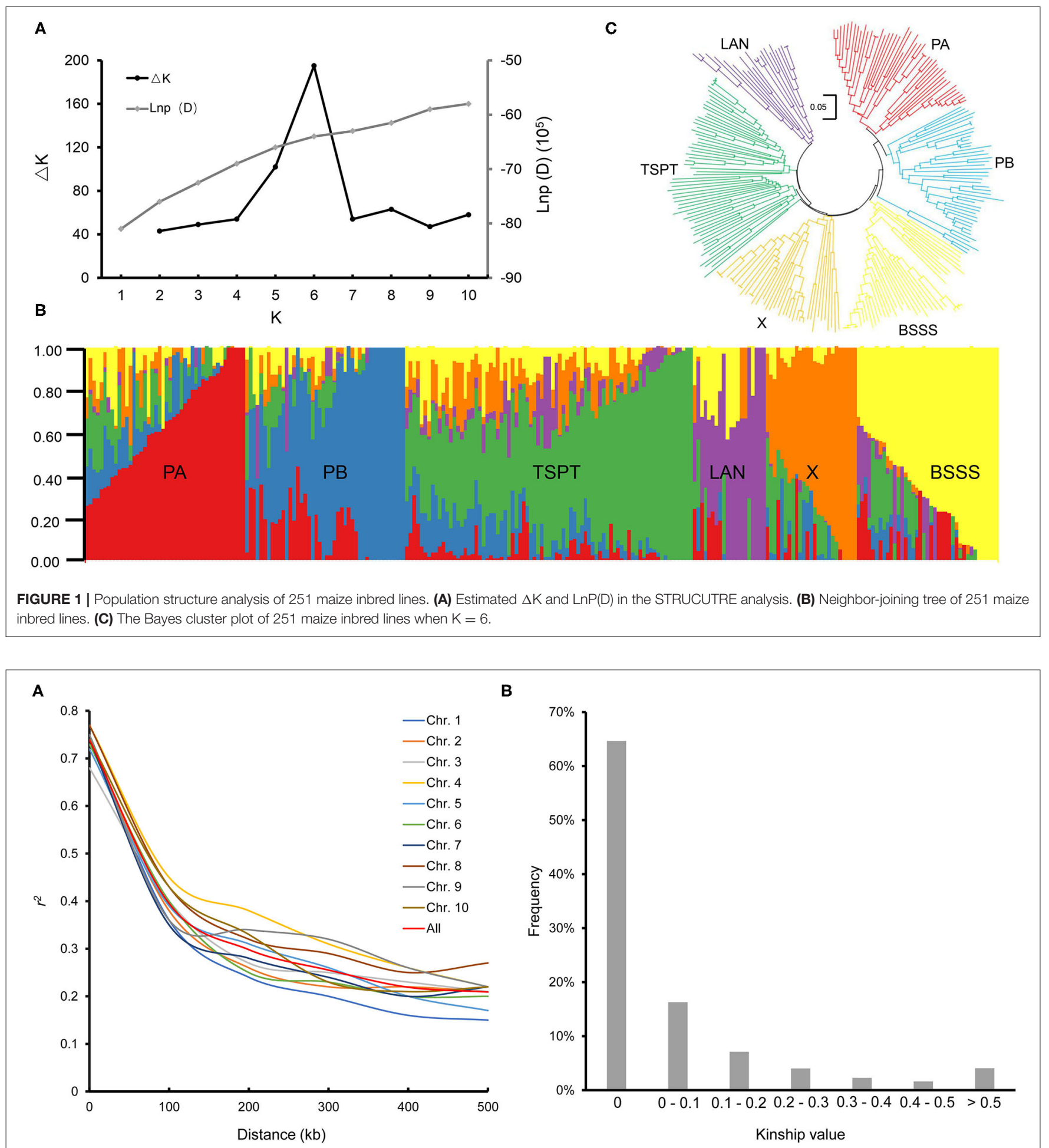

FIGURE 2 | Linkage disequilibrium across the 10 chromosomes (A) and pairwise relative kinship for 251 maize inbred lines (B).

Eleven QTNs were considered to be stable; these were distributed on chromosomes $1,2,3,5,6,7,8$, and 10 (Table 2). Among the 11 stable QTNs, 11 were msQTNs, 1 was esQTNs, and 1 (qKMC2.15) was common between msQTNs and esQTNs. Seven of them overlapped previously reported genomic regions, and the remaining four were putatively novel loci. Only one QTN, qKMC5.6, accounted for more than $10 \%$ of the phenotypic variation 
TABLE 1 | Phenotypic performance, variance component and heritability of KMC.

\begin{tabular}{|c|c|c|c|c|c|c|c|c|}
\hline Env. ${ }^{a}$ & Mean $\pm S^{b}(\%)$ & Range (\%) & Skewness & Kurtosis & W-value & $\sigma_{\mathrm{g}}^{2 c}$ & $\sigma_{\mathrm{ge}}^{2 d}$ & $H^{2}(\%)^{\mathrm{e}}$ \\
\hline Nantong & $34.74 \pm 6.23$ & $18.63-51.13$ & -0.15 & -0.17 & 0.99 & $25.60^{\star \star}$ & & 67.36 \\
\hline Xinxiang & $35.30 \pm 8.60$ & $10.63-52.13$ & -0.29 & -0.29 & 0.99 & $52.11^{\star \star}$ & & 70.42 \\
\hline Sanya & $38.33 \pm 8.03$ & $18.42-53.83$ & -0.56 & -0.42 & 0.97 & $44.63^{\star \star}$ & & 69.73 \\
\hline BLUP & $36.12 \pm 4.79$ & $21.14-47.31$ & 0.19 & -0.42 & 0.99 & $30.20^{\star \star}$ & $9.63^{\star \star}$ & 75.86 \\
\hline
\end{tabular}

${ }^{a}$ Environment.

bStandard deviation.

${ }^{c}$ Variance of genotype.

${ }^{d}$ Variance of genotype $\times$ environment.

${ }^{\mathrm{e}}$ Heritability.

**Significant at $P<0.01$.

(12.41-23.27\%), and it may be a major genetic locus for KMC.

\section{Candidate Genes for KMC}

According to the LD in this association panel (Figure 2A), 63 candidate genes were identified in $100 \mathrm{~kb}$ upstream and downstream of the 11 stable QTNs, and their expression in the kernel varied widely among 11 time points after pollination (Supplementary Table 4). Ten of these genes showed high expression (FPKM $\geq 20$ ), which were marked in bold in Supplementary Table 4, suggesting that they may take part in the loss of kernel moisture. Nine of the genes encode proteins with assigned functions in multiple biological pathways, and the remaining gene encodes a protein of unknown function.

\section{Favorable Allele Mining}

Based on the BLUP values across the three environments, the favorable alleles of 98 QTNs were mined (Supplementary Table 5). The inbred line ID. One hundred and ten harbors the most favorable alleles (69), and the inbred lines ID. Twenty one harbors the smallest favorable alleles (21). At group level, we found a significant negative correlation between the KMC and the number of favorable alleles in each inbred line $\left(R^{2}=0.68\right.$, slope $=-0.41$, intercept $\left.=53.17, P<0.001\right)$ using the linear regression analysis, indicating that pyramiding of these favorable alleles could reduce KMC effectively. There was a significant difference in number of favorable alleles among the six subpopulations; the number of favorable alleles of subgroup $\mathrm{X}$ was the most (48), and that of subgroup PA was the smallest (32) (Figure 4B).

\section{Genomic Selection of KMC}

The average prediction accuracy was $0.12,0.11,0.13$, and 0.17 in NT, XX, SY, and BLUP, respectively, when all 32,853 markers across the entire genome were included in model 1 (Table 3). To obtain optimal prediction accuracy, 12 different marker sets were included as fix effects in model 2. A significant increase in prediction accuracy was obtained when ML-GWAS-derived markers were used: the prediction accuracies based on markers detected by individual ML-GWAS model were almost the same and were approximately 0.34 in NT, 0.53 in XX, 0.29 in SY, and 0.59 in BLUP (Table 3). When all significant markers detected by all six ML-GWAS models were incorporated into the GS model, the prediction accuracy was highest: 0.54 in NT, 0.64 in XX, 0.58 in SY, and 0.76 in BLUP (Table 3).

To explore whether using QTNs co-detected in multiple MLGWAS models could improve prediction accuracy, we conducted GS using QTNs identified in at least two, three, four, five, or six models. Use of the QTNs identified in at least two, three, or four models maintain a relatively high prediction level, but QTNs identified in five or six models provided slight advantage in predicting KMC (Table 3). Seven QTNs were randomly selected from the 44 QTNs identified in at least two models (repeated 5 times) to conducted GS, and the mean of prediction accuracy was relatively low: 0.19 in NT, 0.15 in XX, 0.11 in SY, and 0.19 in BLUP, consistent with the results obtained by GS using QTNs identified in at least five models. This may be due to the smaller QTN numbers, which only explained a small fraction of phenotypic variance.

\section{DISCUSSION}

In this study, the 251 maize inbred lines were sown at three dates according to their growth periods, enabling us to measure KMC of each line over similar periods in each environment. The handheld moisture meter was used to measure $\mathrm{KMC}$, this is a reliable method and has been reported to be useful for evaluating genetic materials for QTL mapping (Sala et al., 2006; Kebede et al., 2016) and GWAS (Zhou et al., 2018; Li et al., 2021). However, we observed a significant genotype by environment interaction and relatively low correlation coefficients (0.44-0.58) among environments. This is typical because temperature, air humidity, and rainfall are uneven across environments, suggesting that genotype-by-environment interactions should be considered during maize breeding. Despite this issue, our aim was to obtain stable genetic loci that make a stable contribution to KMC. As described by Zhang et al. (2019), QTNs identified by multiple models are usually reliable when several ML-GWAS methods are applied to the same dataset. To reduce false positive signals and detect a set number of true positive loci, we considered two types of QTNs to be stable, one is msQTN, which is identified by at least four ML-GWAS models under an uniformed environment, and the other is esQTN, which is identified by in at least two environments and BLUP using the same ML-GWAS model. 


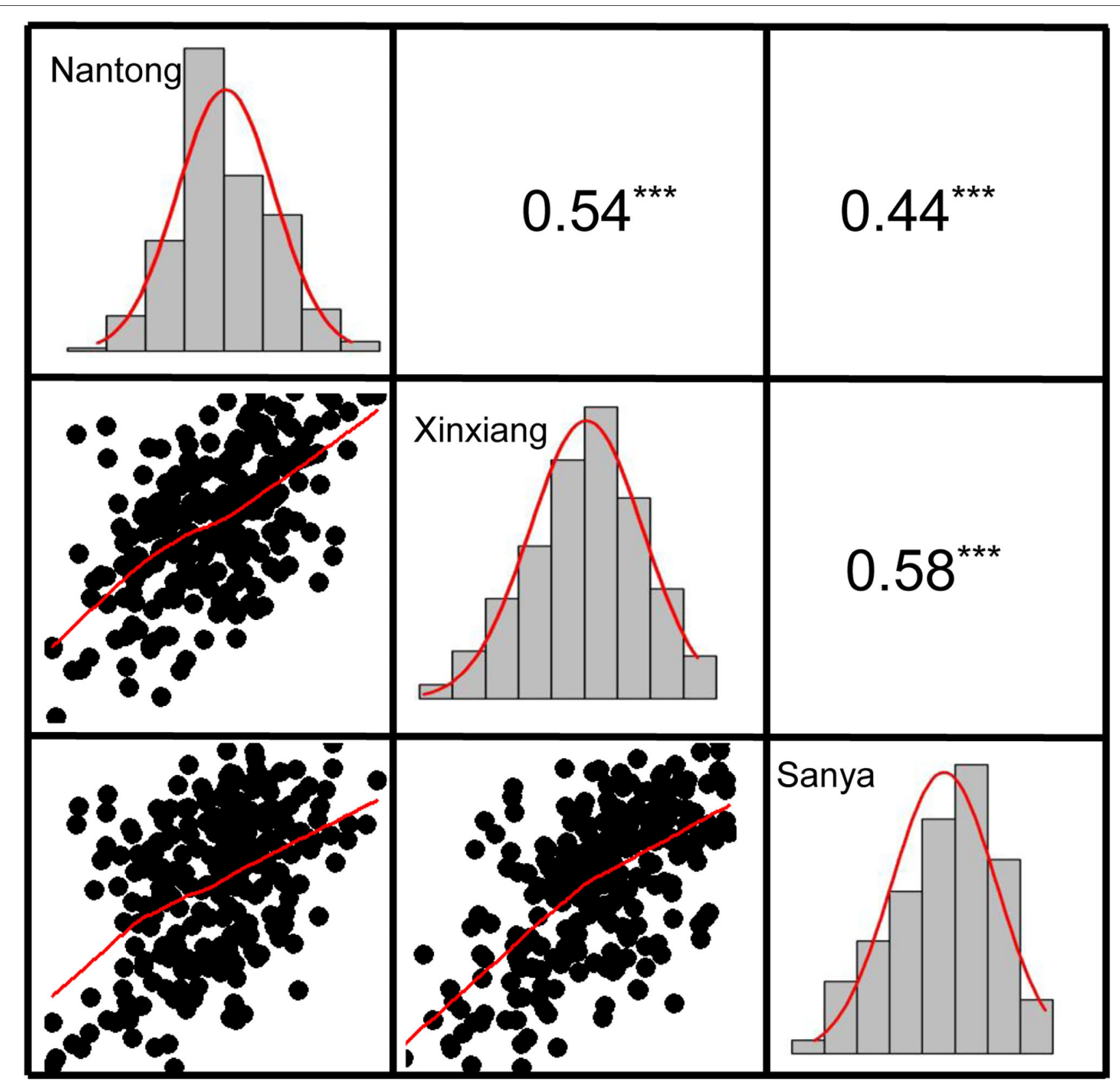

FIGURE 3 | The correlation and frequency distribution of KMC in three environments. The upper panel is correlation coefficients, and the lower panel is scatter plots. The histogram represents the frequency distribution of the trait. ${ }^{\star * *}$ the significance level at $P<0.001$.

Ninety-eight QTNs were identified by six ML-GWAS models in three environments and in BLUP across the three environments; eleven were considered to be stable QTNs. Only one QTN, qKMC5.6, explained more than 10\% of the phenotypic variation, consistent with previous studies in which KMC was mainly controlled by numerous minor-effect genetic loci (Kebede et al., 2016; Song et al., 2017). Of these stable QTNs, 7 were located in genomic regions reported by previous studies, confirming the accuracy of QTN detection by ML-GWAS. Five QTNs ( $q K M C 1.4, q K M C 1.5, q K M C 2.15, q K M C 5.6$, and $q K M C 10.1)$ were located in the meta-QTL regions estimated by Xiang et al. (2012) and Sala et al. (2012). In addition, qKMC3.15 was located in the QTL regions reported by Yin et al. (2020b), and qKMC5.15 overlapped with a QTL region identified by Li et al. (2021). More importantly, four novel genetic loci for KMC were identified in this study.

Candidate gene analysis of the stable QTNs is necessary for further gene cloning and functional verification. To date, only two genes underlying major QTLs for KMC have been identified ( $\mathrm{Li}$ et al., 2021). In this study, 63 candidate genes 

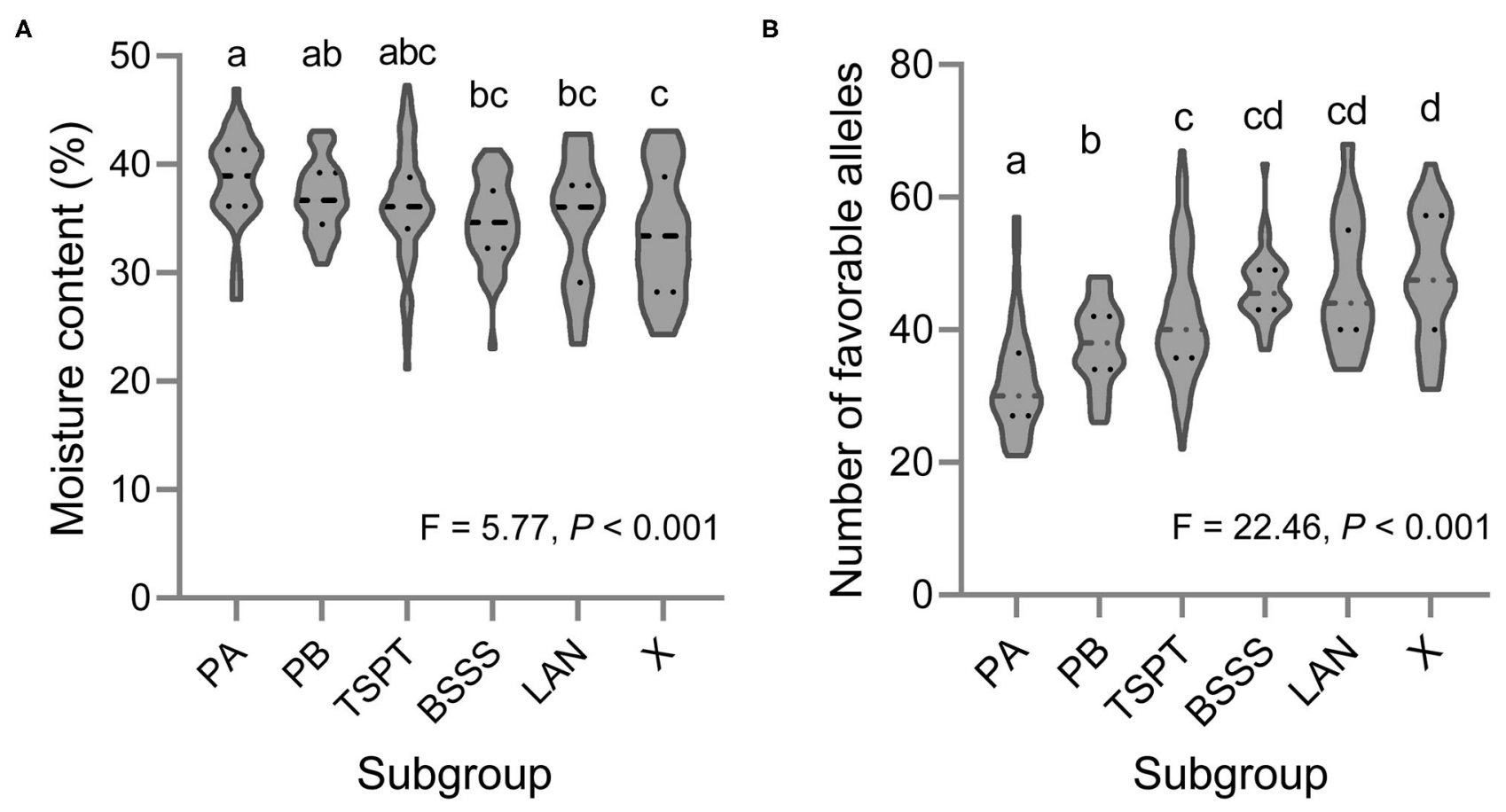

FIGURE 4 | Violin plot of (A) the KMC and (B) the number of favorable alleles for KMC in six subpopulations of this association mapping panel. Different letters indicate significant difference at $P<0.001$ estimated by Student's $t$-test.

were identified surrounding the 11 stable QTNs. Among these genes, 10 were highly expressed in the kernel at different time points after pollination, suggesting that they may potentially affect kernel water loss. We cannot accurately determine which are causal genes associated with $\mathrm{KMC}$ based on the present data. However, four of the candidate genes ( $\mathrm{m} m 00001 \mathrm{d028560}$, Zm00001d005546, Zm00001d014742, and Zm00001d012439) caught our attention.

$Z m 00001 d 028560$, a candidate gene for $q K M C 1.5$, encodes a leucine-rich repeat protein kinase that has been suggested to have an important role in signaling during pathogen recognition (Romeis, 2001; Afzal et al., 2007). Its homologous Arabidopsis gene, FEI 1, participates in cell wall elongation (Xu et al., 2008; Basu et al., 2016). Interestingly, this gene was located in the QTL regions for maize ear rot resistance identified by Butrón et al. (2019), Martin et al. (2012), and Robertson-Hoyt et al. (2007a). Previous studies have reported that KMC is negatively correlated with resistance to maize ear rot (Robertson-Hoyt et al., 2007b; Kebede et al., 2016). Moreover, Xiang et al. (2012) reported 14 pleiotropic meta-QTLs associated with both ear rot resistance and KMC in maize. These results suggest that Zm00001d028560 may be simultaneously related to both KMC and ear rot resistance in maize.

Zm00001d005546, a candidate gene for $q K M C 2.15$, encodes ADP-glucose pyrophosphorylase (AGPase), which provides the nucleotide sugar ADP-glucose and thus constitutes the first step in starch biosynthesis (Slattery et al., 2000; Comparot-Moss and Denyer, 2009). During the maize kernel filling period, AGPase activity and starch synthesis were significantly improved by increasing AGPase expression (Ozbun et al., 1973; Li et al., 2010). In addition, Zm00001d005546 is located in a QTL region related to the maize kernel filling process identified by Yin et al. (2020a). Kernel filling had a notable influence on kernel drying rate before and after physiological maturity in maize (Jia et al., 2020). Therefore, we hypothesize that high expression of Zm00001d005546 may have promoted starch synthesis in the kernel, increasing kernel filling rate, and thereby accelerating kernel dehydration rate before physiological maturity. This may have prolonged the field dehydration time of the kernel and ultimately resulted in low KMC. This explanation also provides a molecular hypothesis for the maize breeding phenomenon in which a hybrid or inbred line with high kernel filling rate generally has low KMC (Johnson and Tanner, 1972; Kang and Zuber, 1989). More experiments are needed to elucidate the function and mechanism of Zm00001d005546.

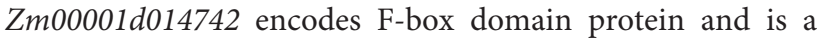
candidate gene for the major QTN, qKMC5.6. Its homologous Arabidopsis gene, AtSKIP31, involves in primary root growth under nitrogen deficiency and regulates the nitrogen utilization efficiencies (Hong et al., 2017). Nitrogen utilization efficiencies are related to grain yield and maturation (Wang W. et al., 2018), which have positive correlation with the KMC (Zhou et al., 2018; Li et al., 2021). Zm00001d012439, a candidate gene for the novel QTN, $q K M C 8.3$, encodes histone H4, which may affect gene transcription activity through histone modification (Heintz, 1991). 
TABLE 2 | Stable QTNs for KMC co-detected by at least four models under a uniform environment or in at least two environments and BLUP using the same ML-GWAS model.

\begin{tabular}{|c|c|c|c|c|c|c|c|c|}
\hline QTN & Marker & Chr. a & Position (bp) & LOD & $R^{2}(\%)^{\mathrm{b}}$ & Modelc & Environment ${ }^{d}$ & References \\
\hline qKMC1.4 & AX-86284737 & 1 & 38082648 & $5.06-9.07$ & $3.14-7.37$ & $1,2,3,4,5,6$ & $X X, B L U P$ & $\begin{array}{l}\text { Sala et al., 2012; } \\
\text { Xiang et al., } 2012\end{array}$ \\
\hline qKMC1.5 & AX-86259253 & 1 & 39246603 & $3.50-9.41$ & $3.02-7.66$ & $1,2,3,4,5,6$ & NT, BLUP & $\begin{array}{l}\text { Sala et al., 2012; } \\
\text { Xiang et al., } 2012\end{array}$ \\
\hline qKMC1.18 & AX-86266353 & 1 & 297863807 & $4.69-6.06$ & $3.13-7.16$ & $1,2,4,5$ & $X X, B L U P$ & \\
\hline qKMC2.15 & AX-116874459 & 2 & 178270600 & $5.15-7.42$ & 2.19-6.23 & $1,3,4,5,6$ & NT, XX, BLUP & Xiang et al., 2012 \\
\hline qКМСЗ.3 & AX-86264182 & 3 & 5148837 & 4.69-7.95 & $3.46-9.74$ & $1,2,4,5,6$ & $X X, B L U P$ & \\
\hline qKMC3.15 & AX-116872692 & 3 & 229667802 & $3.97-5.94$ & 2.33-6.89 & $1,2,3,4,5,6$ & $X X, B L \cup P$ & Yin et al., 2020b \\
\hline qKMC5.6 & $A X-86314969$ & 5 & 61236323 & $4.21-7.84$ & $12.41-23.27$ & $1,2,4,5,6$ & NT & Xiang et al., 2012 \\
\hline qKMC5.15 & AX-86282179 & 5 & 217125252 & $3.23-10.12$ & $1.12-6.48$ & $1,2,4,6$ & NT, BLUP & Li et al., 2021 \\
\hline qKMC6.7 & $A X-86294630$ & 6 & 163230474 & $3.41-7.67$ & $3.29-7.93$ & $1,3,4,6$ & SY & \\
\hline qKMC8.3 & AX-86297230 & 8 & 174417551 & $3.09-5.97$ & $2.45-3.89$ & $2,3,4,5$ & SY, BLUP & \\
\hline qKMC10.1 & $A X-86257470$ & 10 & 10313586 & $3.19-9.60$ & $1.86-9.81$ & $1,2,3,4,5,6$ & $X X$, BLUP & Xiang et al., 2012 \\
\hline
\end{tabular}

${ }^{a}$ Chromosome.

${ }^{b}$ Phenotypic variation explained.

c1: mrMLM, 2: FASTmrMLM, 3: FASTMrEMMA, 4: PLARMEB, 5: PKWmEB, 6: ISIS EM-BLASSO.

${ }^{d} N T$, Nantong; XX, Xinxiang; SY, Sanya; BLUP, best linear unbiased prediction.

In this study, 251 maize inbred lines were divided into six subgroups, that was, PA, PB, TSPT, BASSS, LAN and X. Among these six subgroups, Subgroup PA had highest KMC and the least number of favorable alleles for the KMC, however, Subgroup PA contained multiple elite inbred lines, such as Ye478 and Zheng58, and have played an important role in maize breeding in China over the last 40 years (Li and Wang, 2010). Subgroup X had lowest KMC and the greatest number of favorable alleles for the KMC, and has being gradually applied in maize breeding in China (Zhao et al., 2018). This phenomenon is mainly due to the changes of maize breeding goals at different periods in China. Before 2010, maize harvesting mainly relied on manual. Farmers preferred to plant the varieties with large ear under low density condition to ensure yield. Chinese maize breeders increased yields by extending the growth period. Subgroup PA had the characteristics of large ears and long growth period, which was in line with the breeding goals at that time (Li and Wang, 2010). Maize inbred line or hybrid with large ear and long growth period tended to high KMC (Zhou et al., 2018; Li et al., 2021). In recent years, with the development of agricultural modernization, mechanical harvesting of grain is the developing direction of maize production ( $\mathrm{Li}$ et al., 2017). Chinese maize breeders have increasingly concentrated on the KMC. Thus, a new heterosis group, Subgroup X, was breed. Subgroup X had short growth period and low KMC, which was suitable for mechanical harvesting of maize grain (Zhao et al., 2018).

With advances in sequencing technology and reduction of testing costs, GS has been widely implemented in plant breeding. Fitting GS models need to face the fact that the number of markers $(p)$ far exceeds the number of individuals $(n)$ (de los Campos et al., 2013). Consequently, when a GS model that considers the additive effect of each marker is fitted to such large $p$ and small $n$ data, there will be an infinite number of maximum likelihood estimates of these effects (Gianola, 2013). rrBLUP incorporates all marker information to predict an individual genomic estimated value while simultaneously implementing a penalization function to restrict the values that each marker predicted additive contributions can equal, which is an effective model to overcome this issue (Meuwissen et al., 2001). In this study, the $p$ is 32,853 , while the $n$ is only 251 . Thus, rrBLUP was selected to conduct GS. When using 32,853 markers across the entire genome, we obtained a lower prediction accuracy $(0.11-$ $0.17)$. However, higher prediction levels were easily attained when using the ML-GWAS-derived markers included as fix effects. The prediction accuracy was still high $(0.26-0.56)$ when only 16 stable markers identified by at least four models were included. Similar findings were reported for maize kernel row number (An et al., 2020), resistance to maize southern leaf blight and gray leaf spot (Bian and Holland, 2017), and maize lowphosphorus tolerance (Xu et al., 2018). Therefore, using a small set of markers identified by multiple ML-GWAS methods as fixed effects in an rrBLUP model is a powerful tool for KMC prediction in maize molecular breeding and can effectively save time and costs.

\section{CONCLUSIONS}

Ninety-eight QTNs for KMC were identified using six MLGWAS models in three environments and BLUP across three environments. Eleven QTNs were considered to be stable. Seven stable QTNs corresponded to previously reported QTL regions, whereas the remaining four were putatively novel loci. Sixtythree candidate genes were identified within LD blocks of the 11 stable QTNs. Among these candidates, 10 may potentially affect the loss of water from the maize kernel. High prediction levels were easily reached when the KMC-associated markers were included as fixed effects in GS. The best strategy was to 
TABLE 3 | The prediction accuracy of the KMC when using markers in different ML-GWAS models in three environments and BLUP.

\begin{tabular}{|c|c|c|c|c|c|c|c|c|c|c|c|c|c|c|c|c|}
\hline \multirow[t]{2}{*}{ Marker set ${ }^{a}$} & \multicolumn{4}{|c|}{ Nantong } & \multicolumn{4}{|c|}{ Xinxiang } & \multicolumn{4}{|c|}{ Sanya } & \multicolumn{4}{|c|}{ Best linear unbiased prediction } \\
\hline & $\boldsymbol{R}^{2 \mathrm{~b}}$ & Slope & Intercept & $\mathrm{RMSE}^{\mathrm{C}}$ & $R^{2}$ & Slope & Intercept & RMSE & $R^{2}$ & Slope & Intercept & RMSE & $R^{2}$ & Slope & Intercept & RMSE \\
\hline Genome & 0.12 & 0.83 & 5.99 & 5.84 & 0.13 & 0.90 & 3.45 & 7.97 & 0.11 & 0.81 & 6.07 & 7.84 & 0.17 & 0.85 & 5.20 & 4.35 \\
\hline mrMLM & 0.34 & 0.99 & 0.15 & 5.02 & 0.53 & 0.99 & 0.32 & 5.86 & 0.29 & 0.97 & 0.98 & 6.75 & 0.59 & 1.00 & 0.01 & 3.05 \\
\hline FASTmrMLM & 0.34 & 0.99 & 0.37 & 5.02 & 0.53 & 0.99 & 0.28 & 5.86 & 0.29 & 0.98 & 0.77 & 6.75 & 0.59 & 1.00 & 0.12 & 3.06 \\
\hline FASTmrEMMA & 0.34 & 0.99 & 0.19 & 5.01 & 0.53 & 1.00 & 0.19 & 5.85 & 0.29 & 0.98 & 0.76 & 6.73 & 0.59 & 1.00 & 0.05 & 3.06 \\
\hline PLARmEB & 0.34 & 0.99 & 0.45 & 5.02 & 0.53 & 0.99 & 0.33 & 5.86 & 0.29 & 0.97 & 0.96 & 6.75 & 0.59 & 1.00 & 0.12 & 3.05 \\
\hline PKWmEB & 0.34 & 0.99 & 0.23 & 5.02 & 0.53 & 0.99 & 0.32 & 5.85 & 0.29 & 0.97 & 1.14 & 6.76 & 0.59 & 1.00 & 0.08 & 3.05 \\
\hline ISIS EM-BLASSO & 0.34 & 0.99 & 0.31 & 5.01 & 0.53 & 0.99 & 0.26 & 5.87 & 0.29 & 0.97 & 1.25 & 6.75 & 0.59 & 1.00 & 0.13 & 3.06 \\
\hline All models & 0.54 & 1.01 & -0.20 & 4.21 & 0.64 & 1.00 & -0.06 & 5.12 & 0.58 & 1.02 & -0.60 & 5.16 & 0.76 & 1.01 & -0.29 & 2.32 \\
\hline C2 & 0.46 & 1.01 & -0.31 & 4.55 & 0.60 & 1.00 & -0.03 & 5.42 & 0.42 & 0.99 & 0.43 & 6.06 & 0.69 & 1.00 & -0.05 & 2.64 \\
\hline C3 & 0.47 & 1.00 & -0.05 & 4.48 & 0.54 & 0.99 & 0.33 & 5.81 & 0.33 & 0.98 & 0.33 & 6.52 & 0.65 & 0.99 & 0.05 & 2.81 \\
\hline C4 & 0.42 & 1.00 & 0.03 & 4.72 & 0.48 & 1.00 & 0.09 & 6.19 & 0.26 & 0.98 & 0.82 & 6.87 & 0.56 & 1.00 & 0.10 & 3.17 \\
\hline C5 & 0.19 & 0.98 & 0.86 & 5.59 & 0.20 & 0.99 & 0.51 & 7.42 & 0.14 & 0.95 & 1.21 & 7.81 & 0.22 & 0.96 & 1.10 & 4.21 \\
\hline C6 & 0.15 & 0.92 & 2.80 & 6.03 & 0.16 & 0.97 & 0.86 & 8.06 & 0.13 & 0.88 & 0.86 & 7.89 & 0.19 & 0.96 & 1.36 & 4.53 \\
\hline
\end{tabular}

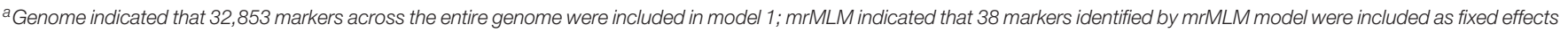

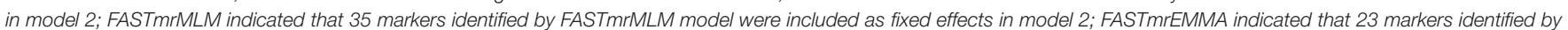

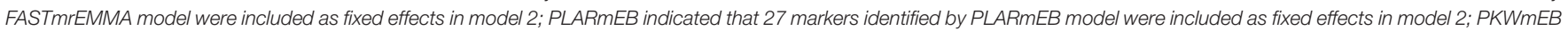

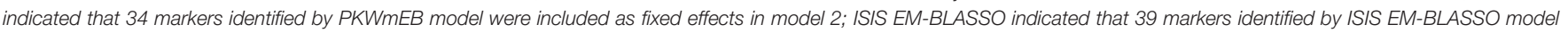

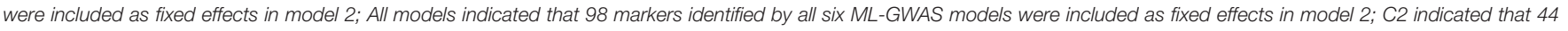

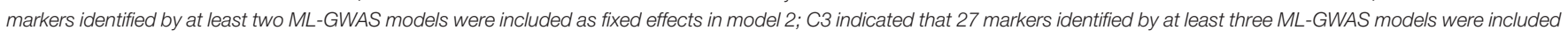

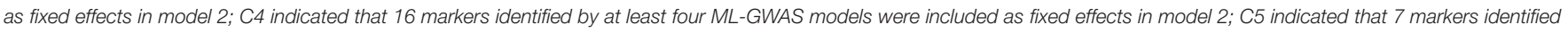

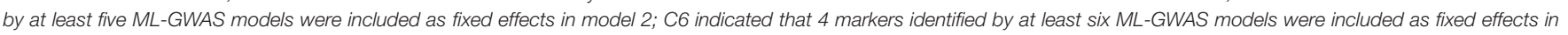
model 2.

${ }^{b}$ Coefficient of determination.

cSquare root of the mean square error.

integrate all KMC-associated markers identified by all six MLGWAS models. These results facilitate our understanding of the genetic basis of KMC and provide useful information for the reduction of KMC in maize breeding.

\section{DATA AVAILABILITY STATEMENT}

The original contributions presented in the study are included in the article/Supplementary Material, further inquiries can be directed to the corresponding author/s.

\section{AUTHOR CONTRIBUTIONS}

GZ and DH designed the experiment and wrote the manuscript. GZ, YM, LX, GC, ZZ, XS, HZ, HL, MS, and DH performed the experiments and collected the phenotypic data. GZ and QZ analyzed the genotypic and phenotypic data. All authors read and approved the final manuscript.

\section{FUNDING}

This study was supported by the Jiangsu Agriculture Science and Technology Innovation Fund, China [CX(19)3049], the Scientific and Technological Project of Jiangsu Province, China (BE2018325), and the Earmarked Fund for Jiangsu Agricultural Industry Technology System, China.

\section{ACKNOWLEDGMENTS}

The authors would like to thank TopEdit (www.topeditsci.com) for linguistic assistance during preparation of this manuscript.

\section{SUPPLEMENTARY MATERIAL}

The Supplementary Material for this article can be found online at: https://www.frontiersin.org/articles/10.3389/fpls.2021. 697688/full\#supplementary-material

Supplementary Figure 1 | Manhattan (left) and quartile-quartile (right) plots of ML-GWAS results using mrMLM model. The four circles of Manhattan plot from inside to outside show the result in Nantong (NT), Xinxiang (XX), Sanya (SY), and best linear unbiased prediction (BLUP), respectively.

Supplementary Figure 2 | Manhattan (left) and quartile-quartile (right) plots of ML-GWAS results using FASTmrMLM model. The four circles of Manhattan plot from inside to outside show the result in Nantong (NT), Xinxiang (XX), Sanya (SY), and best linear unbiased prediction (BLUP), respectively.

Supplementary Figure $\mathbf{3}$ | Manhattan (left) and quartile-quartile (right) plots of ML-GWAS results using FASTmrEMMA model. The four circles of Manhattan plot from inside to outside show the result in Nantong (NT), Xinxiang (XX), Sanya (SY), and best linear unbiased prediction (BLUP), respectively.

Supplementary Figure $\mathbf{4}$ | Manhattan (left) and quartile-quartile (right) plots of ML-GWAS results using PKWmEB model. The four circles of Manhattan plot from inside to outside show the result in Nantong (NT), Xinxiang (XX), Sanya (SY), and best linear unbiased prediction (BLUP), respectively.

Supplementary Figure $\mathbf{5}$ | Manhattan (left) and quartile-quartile (right) plots of SL-GWAS results using MLM model. The four circles of Manhattan plot from 
inside to outside show the result in Nantong (NT), Xinxiang (XX), Sanya (SY), and best linear unbiased prediction (BLUP), respectively.

Supplementary Table 1 | The origin or pedigree, subpopulation, sowing data, and phenotype of 251 maize inbred lines used in this study.

Supplementary Table 2 | Analysis of variance for KMC across three environments.

\section{REFERENCES}

Afzal, A. J., Wood, A. J., and Lightfoot, D. A. (2007). Plant receptor-like serine threonine kinases: roles in signaling and plant defense. Mol. Plant Microbe Interact. 21, 507-517. doi: 10.1094/MPMI-21-5-0507

An, Y. X., Chen, L., Li, Y. X., Li, C. H., Shi, Y. S., Zhang, D. F., et al. (2020). Genome-wide association studies and whole-genome prediction reveals the genetic architecture of KRN in maize. BMC Plant Biol. 20:490. doi: 10.1186/s12870-020-02676-x

Austin, D. F., Lee, M., Veldboom, L. R., and Hallauer, A. R. (2000). Genetic mapping in maize with hybrid progeny across testers and generations: grain yield and grain moisture. Crop Sci. 40, 30-39. doi: 10.2135/cropsci2000. 40130x

Basu, S., Tian, L., Debrosse, T., Poirier, E., Emch, K., Herock, H., et al. (2016). Glycosylation of a fasciclin-like arabinogalactan-protein (SOS5) mediates root growth and seed mucilage adherence via a cell wall receptorlike kinase (FEI1/FEI2) pathway in Arabidopsis. PLOS ONE 11:e0145092. doi: 10.1371/journal.pone.0145092

Beavis, W. D., Smith, O. S., Grant, D., and Fincher, R. (1994). Identification of quantitative trait loci using a small sample of topcrossed and $\mathrm{F}_{4}$ progeny from maize. Crop Sci. 34, 882-896. doi: 10.2135 /cropsci1994.0011183X003400040010x

Bian, Y., and Holland, J. B. (2017). Enhancing genomic prediction with genomewide association studies in multiparental maize populations. Heredity 118, 585-593. doi: 10.1038/hdy.2017.4

Blanc, G., Charcosset, A., Mangin, B., Gallais, A., and Moreau, L. (2006). Connected populations for detecting quantitative trait loci and testing for epistasis: an application in maize. Theor. Appl. Genet. 113, 206-224. doi: 10.1007/s00122-006-0287-1

Bradbury, P. J., Zhang, Z., Kroon, D. E., Casstevens, T. M., Ramdoss, Y., and Buckler, E. S. (2007). TASSEL: software for association mapping of complex traits in diverse samples. Bioinformatics 23, 2633-2635. doi: 10.1093/bioinformatics/btm308

Brooking, I. P. (1990). Maize ear moisture during grain-filling, and its relation to physiological maturity and grain-drying. Field Crops Res. 23, 55-68. doi: 10.1016/0378-4290(90)90097-U

Butrón, A., Santiago, R., Cao, A., Samayoa, L. F., and Malvar, R. A. (2019). QTLs for resistance to Fusarium ear rot in a multi-parent advanced generation inter-cross (MAGIC) of maize population. Plant Dis. 103, 897-904. doi: 10.1094/PDIS-09-18-1669-RE

Capelle, V., Remoué, C., Moreau, L., Reyss, A., Mahé, A., Massonneau, A., et al. (2010). QTLs and candidate genes for desiccation and abscisic acid content in maize kernels. BMC Plant Biol. 10:2. doi: 10.1186/1471-2229-10-2

Comparot-Moss, S., and Denyer, K. (2009). The evolution of the starch biosynthetic pathways in cereals and other grasses. J. Exp. Bot. 60, 2481-2492. doi: $10.1093 / \mathrm{jxb} / \mathrm{erp} 141$

de los Campos, G., Hickey, J. M., Pong-Wong, R., Daetwyler, H. D., and Calus, M. P. L. (2013). Whole-genome regression and prediction methods applied to plant and animal breeding. Genetics 193, 327-345. doi: 10.1534/genetics.112.143313

Endelman, J. B. (2011). Ridge regression and other kernels for genomic selection with $\mathrm{R}$ package rrBLUP. Plant Genome 4, 250-255. doi: 10.3835/plantgenome2011.08.0024

Evanno, G., Regnaut, S., and Goudet, J. (2005). Detecting the number of clusters of individuals using the software STRUCTURE: a simulation study. Mol. Ecol. 14, 2611-2620. doi: 10.1111/j.1365-294X.2005.02553.X

Frascaroli, E., Canè, M., Landi, P., Pea, G., Gianfranceschi, L., Villa, M., et al. (2007). Classical genetic and quantitative trait loci analyses of
Supplementary Table 3 | The significant QTNs for KMC identified by MLM and six ML-GWAS models in this study.

Supplementary Table 4 | Candidate genes around the 11 stable QTNs for KMC and their FPKM expression data in the whole kernle of B73 at different days after pollination (DAP).

Supplementary Table 5 | The distribution of favorable alleles for 98 QTNs in 251 maize inbred lines.

heterosis in a maize hybrid between two elite inbred lines. Genetics 176:625 doi: 10.1534/genetics.106.064493

Gianola, D. (2013). Priors in whole-genome regression: the Bayesian alphabet returns. Genetics 194, 573-596. doi: 10.1534/genetics.113.151753

Hardy, O. J., and Vekemans, X. (2002). SPAGeDi: a versatile computer program to analyse spatial genetic structure at the individual or population levels. Mol. Ecol. Res. 2, 618-620. doi: 10.1046/j.1471-8286.2002.00305.x

Heintz, N. (1991). The regulation of histone gene expression during the cell cycle. Biochim. Biophys. Acta 1088, 327-339. doi: 10.1016/0167-4781(91)90122-3

Ho, J., Mccouch, S., and Smith, M. (2002). Improvement of hybrid yield by advanced backcross qtl analysis in elite maize. Theor. Appl. Genet. 105:440. doi: 10.1007/s00122-002-0945-X

Hong, J. P., Adams, E., Yanagawa, Y., Matsui, M., and Shin, R. (2017). AtSKIP18 and AtSKIP31, F-box subunits of the SCF E3 ubiquitin ligase complex, mediate the degradation of 14-3-3 proteins in Arabidopsis. Biochem. Bioph. Res. Co. 485, 174-180. doi: 10.1016/j.bbrc.2017.02.046

Jia, T. J., Wang, L. F., Li, J. J., Ma, J., Cao, Y. Y., Lübberstedt, T., et al. (2020). Integrating a genome-wide association study with transcriptomic analysis to detect genes controlling grain drying rate in maize (Zea may L.). Theor. Appl. Genet. 133, 623-634. doi: 10.1007/s00122-019-03492-0

Johnson, D. R., and Tanner, J. W. (1972). Calculation of the rate and duration of grain filling in corn (Zea mays L.). Crop Sci. 12, 485-486. doi: 10.2135/cropsci1972.0011183X001200040028x

Kang, M. S., and Zuber, M. S. (1989). Combining ability for grain moisture, husk moisture, and maturity in maize with yellow and white endosperms. Crop Sci. 29, 689-692. doi: 10.2135/cropsci1989.0011183X002900030030x

Kebede, A. Z., Woldemariam, T., Reid, L. M., and Harris, L. J. (2016). Quantitative trait loci mapping for Gibberella ear rot resistance and associated agronomic traits using genotyping-by-sequencing in maize. Theor. Appl. Genet. 129, 17-29. doi: 10.1007/s00122-015-2600-3

Knapp, S. J., Stroup, W. W., and Ross, W. M. (1985). Exact confidence intervals for heritability on a progeny mean basis. Crop Sci. 25, 192-194. doi: 10.2135/cropsci1985.0011183X002500010046x

Kumar, S., Stecher, G., Li, M., Knyaz, C., and Tamura, K. (2018). MEGA X: molecular evolutionary genetics analysis across computing platforms. Mol. Biol. Evol. 35, 1547-1549. doi: 10.1093/molbev/msy096

Li, L. L., Lei, X. P., Xie, R. Z., Wang, K. R., Hong, P., Zhang, F. G., et al. (2017). Analysis of influential factors on mechanical grain harvest quality of summer maize. Sci. Agric. Sin. 50, 2044-2051. doi: 10.3864/j.issn.0578-1752.2017.11.010

Li, L. L., Xie, R. Z., Fan, P. P., Lei, X. P., Wang, K. R., Hou, P., et al. (2016). Study on dehydration in kernel between Zhengdan958 and Xianyu335. J. Maize Sci. 24, 57-61. doi: 10.13597/j.cnki.maize.science.20160212

Li, N., Zhang, S. J., Zhao, Y. J., Li, B., and Zhang, J. R. (2010). Over-expression of AGPase genes enhances seed weight and starch content in transgenic maize. Planta 233, 241-250. doi: 10.1007/s00425-010-1296-5

Li, W. Q., Yu, Y. H., Wang, L. X., Luo, Y., Peng, Y., Xu, Y. C., et al. (2021). The genetic architecture of the dynamic changes in grain moisture in maize. Plant Biotechnol. J. 19, 1195-1205. doi: 10.1111/pbi.13541

Li, Y., and Wang, T. Y. (2010). Germplasm base of maize breeding in China and formation of foundation parents. J. Maize Sci. 18, 1-8.

Liu, F. H., Wang, K. R., Jian, L., Wang, X. M., Sun, Y. L., Chen, Y. S., et al. (2013). Factors affecting corn mechanically harvesting grain quality. Crops 4, 116-119.

Liu, J. J., Yu, H., Liu, Y. L., Deng, S. N., Liu, Q. C., Liu, B. S., et al. (2020). Genetic dissection of grain water content and dehydration rate related to mechanical harvest in maize. BMC Plant Biol. 20:118. doi: 10.1186/s12870-020-2302-0

Martin, M., Miedaner, T., Schwegler, D. D., Kessel, B., Ouzunova, M., Dhillon, B. S., et al. (2012). Comparative quantitative trait loci mapping for Gibberella ear rot 
resistance and reduced deoxynivalenol contamination across connected maize populations. Crop Sci. 52, 32-43. doi: 10.2135/cropsci2011.04.0214

Melchinger, A. E., Utz, H. F., and Schön, C. C. (1998). Quantitative trait locus (QTL) mapping using different testers and independent population samples in maize reveals low power of QTL detection and large bias in estimates of QTL effects. Genetics 149, 383-403. doi: 10.1093/genetics/149.1.383

Meuwissen, T. H., Hayes, B. J., and Goddard, M. E. (2001). Prediction of total genetic value using genome-wide dense marker maps. Genetics 157, 1819-1829. doi: 10.1093/genetics/157.4.1819

Mihaljevic, R., Utz, H. F., and Melchinger, A. E. (2004). Congruency of quantitative trait loci detected for agronomic traits in testcrosses of five populations of European maize. Crop Sci. 44, 114-124. doi: 10.2135/cropsci2004.1140

Mihaljevic, R., Utz, H. F., and Melchinger, A. E. (2005). No evidence for epistasis in hybrid and per se performance of elite European flint maize inbreds from generation means and qtl analyses. Crop Sci. 45, 2605-2613. doi: $10.2135 /$ cropsci2004.0760

Nei, M. F. (1972). Genetic distance between populations. Am. Nat. 106, 283-292. doi: $10.1086 / 282771$

Ozbun, J. L., Hawker, J. S., Greenberg, E., Lammel, C., and Preiss, J. (1973). Starch synthetase, phosphorylase, ADPglucose pyrophyosphortlase, and UDPglucose pyrophyosphortlase in developing maize kernels. Plant Physiol. 51, 1-5. doi: 10.1104/pp.51.1.1

Pritchard, J. K., Stephens, M., and Donnelly, P. (2000). Infernece of population structure using multilocus gentoype data. Genetics 155, 945-959. doi: 10.1093/genetics/155.2.945

Purcell, S., Neale, B., Todd-Brown, K., Thomas, L., Ferreira, M. A., Bender, D., et al. (2007). PLINK: a tool set for whole-genome association and population-based linkage analysis. Am. J. Hum. Genet. 81, 559-575. doi: 10.1086/519795

Qin, J., Shi, A. N., Song, Q. J., Li, S., Wang, F. M., Cao, Y. D., et al. (2019). Genomicwide association study and genomic selection of amino acid concentrations in soybean seeds. Front. Plant Sci. 10:1445. doi: 10.3389/fpls.2019.01445

Ravelombola, W. S., Qin, J., Shi, A. N., Nice, L., Bao, Y., Lorenz, A., et al. (2019). Genome-wide association study and genomic selection for soybean chlorophyll content associated with soybean cyst nematode tolerance. BMC Genomics 20:904. doi: 10.1186/s12864-019-6275-Z

Reid, L. M., Zhu, X., Morrison, M. J., Woldemariam, T., Voloaca, C., Wu, J., et al. (2010). A non-destructive method for measuring maize kernel moisture in a breeding program. Maydica 55, 163-171. doi: 10.3198/jpr2009.06.0350crmp

Ren, W. L., Wen, Y. J., Dunwell, J. M., and Zhang, Y. M. (2018). pKWmEB: integration of Kruskal-Wallis test with empirical bayes under polygenic background control for multi-locus genome-wide association study. Heredity 120, 208-218. doi: 10.1038/s41437-017-0007-4

Roberston-Hoyt, L. A., Kleinschmidt, C. E., White, D. G., Payne, G. A., Maragos, C. M., and Holland, J. B. (2007b). Relationships of resistance to Fusarium ear rot and Fumonisin contamination with agronomic performance of maize. Crop Sci. 47, 1770-1778. doi: 10.2135/cropsci2006.10.0676

Robertson-Hoyt, L. A., Betrán, J., Payne, G. A., White, D. G., Isakeit, T., Maragos, C. M., et al. (2007a). Relationships among resistances to Fusarium and Aspergillus ear rots and contamination by fumonisin and aflatoxin in maize. Phytopathology 97, 311-317. doi: 10.1094/PHYTO-97-3-0311

Romeis, T. (2001). Protein kinases in the plant defence response. Curr. Opin. Plant Biol. 4, 407-414. doi: 10.1016/S1369-5266(00)00193-X

Sala, R. G., Andrade, F. H., Camadro, E. L., and Cerono, J. C. (2006). Quantitative trait loci for grain moisture at harvest and field grain drying rate in maize (Zea mays L.). Theor. Appl. Genet. 112, 462-471. doi: 10.1007/s00122-005-0146-5

Sala, R. G., Andrade, F. H., and Cerono, J. C. (2012). Quantitative trait loci associated with grain moisture at harvest for line per se and testcross performance in maize: a meta-analysis. Euphytica 185, 429-440. doi: 10.1007/s10681-011-0614-8

Sehgal, D., Rosyara, U., Mondal, S., Singh, R., Poland, J., and Dreisigacker, S. (2020). Incorporating genome-wide association mapping results into genomic prediction models for grain yield and yield stability in CIMMYT spring bread wheat. Front Plant Sci. 11:197. doi: 10.3389/fpls.2020.00197

Slattery, C. J., Kavakli, I. H., and Okita, T. W. (2000). Engineering starch for increased quantity and quality. Trends Plant Sci. 5, 291-298. doi: 10.1016/S1360-1385(00)01657-5
Song, W., Shi, Z., Xing, J. F., Duan, M. X., Su, A. G., Li, C. H., et al. (2017) Molecular mapping of quantitative trait loci for grain moisture at harvest in maize. Plant Breed. 136, 28-32. doi: 10.1111/pbr.12430

Spindel, J. E., Begum, H., Akdemir, D., Collard, B., Redona, E., Jannink, J. L., et al. (2016). Genome-wide prediction models that incorporate de novo GWAS are a powerful new tool for tropical rice improvement. Heredity 116, 395-408. doi: 10.1038/hdy.2015.113

Tamba, C. L., Ni, Y. L., and Zhang, Y. M. (2017). Iterative sure independence screening EM-Bayesian LASSO algorithm for multilocus genome-wide association studies. PLoS Comput. Biol. 13:e1005357. doi: 10.1371/journal.pcbi.1005357

Tamba, C. L., and Zhang, Y. M. (2018). A fast mrMLM algorithm for multi-locus genome-wide association studies. bioRxiv [Preprint]. doi: 10.1101/341784

Wang, S. B., Feng, J. Y., Ren, W. L., Huang, B., Zhou, L., Wen, Y. J., et al. (2016). Improving power and accuracy of genome-wide association studies via a multi-locus mixed linear model methodology. Sci. Rep. 6:19444. doi: $10.1038 /$ srep 19444

Wang, W., Hu, B., Yuan, D. Y., Liu, Y. Q., Che, R. H., Hu, Y. C., et al. (2018). Expression of the nitrate transporter gene OsNRT1.1A/OsNPF6.3 confers high yield and early maturation in rice. Plant Cell 30, 638-651. doi: 10.1105/tpc.17.00809

Wang, X., Xu, Y., Hu, Z. L., and Xu, C. W. (2018). Genomic selection methods for crop improvement: current status and prospects. Crop J. 6, 330-340. doi: 10.1016/j.cj.2018.03.001

Wen, Y. J., Zhang, H., Ni, Y. L., Huang, B., Zhang, J., Feng, J. Y., et al. (2018). Methodological implementation of mixed linear models in multi-locus genome-wide association studies. Brief. Bioinform. 19, 700-712. doi: 10.1093/bib/bbw145

Xiang, K., Reid, L. M., Zhang, Z. M., Zhu, X. Y., and Pan, G. T. (2012). Characterization of correlation between grain moisture and ear rot resistance in maize by QTL meta-analysis. Euphytica 183, 185-195. doi: 10.1007/s10681-011-0440-z

Xu, C., Zhang, H. W., Sun, J. H., Guo, Z. F., Zou, C., Li, W. X., et al. (2018). Genome-wide association study dissects yield components associated with lowphosphorus stress tolerance in maize. Theor. Appl. Genet. 131, 1699-1714. doi: 10.1007/s00122-018-3108-4

Xu, S. L., Rahman, A., Baskin, T. I., and Kieber, J. J. (2008). Two leucine-rich repeat receptor kinases mediate signaling, linking cell wall biosynthesis and ACC synthase in Arabidopsis. Plant Cell 20, 3065-3079. doi: 10.1105/tpc.108. 063354

Xu, Y. B., Liu, X. G., Fu, J. J., Wang, H. W., Wang, J. K., Huang, C. L., et al. (2020). Enhancing genetic grain through genomic selection: from livestock to plants. Plant Comm. 1:100005. doi: 10.1016/j.xplc.2019. 100005

Yin, S. Y., Li, P. C., Xu, Y., Liu, J., Yang, T. T., Wei, J., et al. (2020a). Genetic and genomic analysis of the seed-filling process in maize based on a logistic model. Heredity 124, 122-134. doi: 10.1038/s41437-0190251-X

Yin, S. Y., Liu, J., Yang, T. T., Li, P. C., Xu, Y., Fang, H. M., et al. (2020b). Genetic analysis of the seed dehydration process in maize based on a logistic model. Crop J. 8, 182-193. doi: 10.1016/j.cj.2019.06.011

Zhang, J., Feng, J. Y., Ni, Y. L., Wen, Y. J., Niu, Y., Tamba, C. L., et al. (2017). pLARmEB: integration of least angle regression with empirical bayes for multi-locus genome-wide association studies. Heredity 118, 517-524. doi: 10.1038/hdy.2017.8

Zhang, J., Zhang, F. Q., Tang, B. J., Ding, Y., Xia, L. K., Qi, J. S., et al. (2020). Molecular mapping of quantitative trait loci for grain moisture at harvest and field grain drying rat in maize (Zea mays L.). Physiol. Plant. 169, 64-72. doi: $10.1111 /$ ppl.13048

Zhang, Y. L., Liu, P., Zhang, X. X., Zheng, Q., Chen, M., Ge, F., et al. (2018). Multi-locus genome-wide association study reveals the genetic architecture of stalk lodging resistance-related traits in maize. Front. Plant Sci. 9:611. doi: $10.3389 /$ fpls.2018.00611

Zhang, Y. M., Jia, Z., and Dunwell, J. M. (2019). Editorial: the application of new multi-locus GWAS methodologies in the genetic dissection of complex traits. Front. Plant Sci. 10:100. doi: 10.3389/fpls.2019.00100 
Zhao, J. R., Li, C. H., Song, W., Wang, Y. D., Zhang, R. Y., Wang, J. D., et al. (2018). Genetic diversity and population structure of important Chinese maize breeding germplasm revealed by SNP-Chips. Sci. Agric. Sin. 51, 626-634. doi: 10.3864/j.issn.0578-1752.2018.04.003

Zhou, G. F., Hao, D. R., Chen, G. Q., Lu, H. H., Shi, M. L., Mao, Y. X., et al. (2016). Genome-wide association study of the husk number and weight in maize (Zea mays L.). Euphytica 210, 195-205. doi: 10.1007/s10681-016$1698-y$

Zhou, G. F., Hao, D. R., Xue, L., Chen, G. Q., Lu, H. H., Zhang, Z. L., et al. (2018). Genome-wide association study of kernel moisture content at harvest stage in maize. Breed. Sci. 68, 622-628. doi: 10.1270/jsbbs. 18102

Zhou, G. F., Mao, Y. X., Xue, L., Chen, G. Q., Lu, H. H., Shi, M. L., et al. (2020). Genetic dissection of husk number and length across multiple environments and fine-mapped of a major-effect QTL for husk number in maize. Crop J. 8, 1071-1080. doi: 10.1016/j.cj.2020.03.009

Conflict of Interest: The authors declare that the research was conducted in the absence of any commercial or financial relationships that could be construed as a potential conflict of interest.

Copyright (c) 2021 Zhou, Zhu, Mao, Chen, Xue, Lu, Shi, Zhang, Song, Zhang and Hao. This is an open-access article distributed under the terms of the Creative Commons Attribution License (CC BY). The use, distribution or reproduction in other forums is permitted, provided the original author(s) and the copyright owner(s) are credited and that the original publication in this journal is cited, in accordance with accepted academic practice. No use, distribution or reproduction is permitted which does not comply with these terms. 